\title{
Assessment and Management of Pain, Alignment, Strength and Stability (PASS) in Patellofemoral Pain and Low Back Pain
}

\author{
Alex Mbuli ${ }^{1,2}$, Ambreen Chohan ${ }^{1}$, Jessica Janssen ${ }^{1}$, Olivia Greenhalgh ${ }^{1}$, Lauren Haworth ${ }^{1}$, Hannah Shore ${ }^{1}$, \\ Mairi Olivier ${ }^{1}$, Hazel Roddam ${ }^{1}$, Louise Anne Connell ${ }^{1}$, Jim Richards ${ }^{1}$
}

${ }^{1}$ Allied Health Research unit, University of Central Lancashire, UK

${ }^{2}$ Sport, Exercise \& Health Sciences, Edinburgh Napier University, UK

\begin{abstract}
Clinical assessment and management of musculoskeletal conditions of different joints may be broken down into considerations of Pain, Alignment, Strength and Stability (PASS). In recent years these factors have allowed a systematic approach and has enabled the development in our understanding of clinical subgroups, which enable targeted or stratified care. This paper considers the use of the PASS concept to determine the most appropriate treatment and interventions, specifically when considering treatment of two common musculoskeletal conditions, patellofemoral pain and low back pain.
\end{abstract}

\section{KEY WORDS}

Pain, Alignment, Strength, Stability, Musculoskeletal Assessment

\section{INTRODUCTION}

The clinical assessment and management of musculoskeletal conditions of different joints may be broken down into considerations of Pain, Alignment, Strength and Stability (PASS), which may be used to assess treatment and interventions. This provides a framework that encourages the practitioner and researcher to address these four factors when considering a treatment for a specific pathology. Each of the factors outlined below need to be considered, as these help to identify the specific needs of the patient, which can be used to target specific aspects and outcomes of the condition and provides a patient centred approach.

Pain may result from an injury or an ongoing condition such as mechanical low back pain (MLBP) or patellofemoral pain (PFP), this can result in a reduction in activity and can affect an individual's quality of life. According to the Health and Safety Executive's (HSE) annual statistics (1), in the United Kingdom 2.2 million working days are lost as a result of back disorders and 1.7 million as a result of work related lower limb disorders. By addressing this aspect of PASS, the impact of pain may be minimised through a clinically significant reduction in pain. This in turn may lead to patients moving with greater ease and being able to return to activities of daily living or sports. When considering alignment we often consider malalignment, or the lack of control of alignment of specific joints within the body. This can have devastating results on an individual's participation in activities of daily living. By observing deficits in movement patterns of joints and surrounding structures, it may be possible to use specific interventions to target and correct such deficits; these in turn will then have a positive impact on pain and wellbeing. Richards et al (2) showed that by using a targeted intervention such as knee bracing in osteoarthritis patients improvements in function, loading and propulsive forces can be made. Strength is also a key aspect that allows practitioners to determine deficits in force production. By strengthening muscular structures through increased physical activity and targeted exercise regimes a reduction has been seen in pain and disability that has been associated with knee arthritis (3). Weakness in a structure could lead to compensations, which could negatively impact or even cause injury at another site. However, strength should not just be considered in terms of maximal output, but should be put in context as to the optimal force for the structures being assessed, as over strengthening could lead to a muscle imbalance or joint overload. Such an imbalance in strength can result in deficits in 
musculoskeletal stability, however these may not be just mechanical, and proprioceptive or control deficits may also be responsible.

The Medical Research Council (MRC) guidelines for complex interventions (4) state that researchers should be clear about the aim when developing a study. By framing the research question around the elements of PASS, the researcher or practitioner can ensure that they are establishing the specific area, or areas of a condition, which they are trying to address. The use of the PASS framework allows researchers to consider the implementation of their findings. The MRC guidelines (2019) asks whether the findings of a study can be widely implemented if the results are favourable. By addressing the factors outlined in PASS, the route to implementation can be mapped, as this addresses specific functional deficits, which in turn can be mapped to specific interventions strategies. In addition, the MRC guidelines (2019) propose that the results be accessible to decision-makers, which includes patients, who are key to the decision making process. Using PASS, patients should be able to better comprehend what the treatments or interventions are trying to target.

\section{THE USE OF PASS IN THE MANAGEMENT OF LOW BACK PAIN}

Eighty percent of adults experience Low Back Pain (LBP) at some point in their adult life (5). LBP is a costly musculoskeletal disorder, often relating to poor posture and movement habits, and caused by an imbalance in the supporting structures of the (6). LBP patients often struggle to move freely, and activities of daily living, sleep and work are often hindered as a result of pain $(7,8,9,10)$. Highly significant relationships have been shown in the literature between LBP and quality of sleep, with reports of $55 \%$ increase in restless/light sleep following the onset of pain (11). However, LBP is a broad term often used to cover a multitude of chronic and acute, muscular, mechanical and neurological disorders. It has been shown that a large majority (80-90\%) of individuals will recover within 12 weeks (12), however permanent disability accounts for $5-15 \%$ of (13). This has a huge potential economic effect on annual direct healthcare costs, which has previously been estimated to reach $£ 1632$ million in the UK (14). Therefore, correct clinical diagnosis is key to the effective treatment and rehabilitation. As a result, a number of measures and tools commonly used in clinical practice have been included within research studies published to date. These help determine the effectiveness of different interventions in the management of LBP.
Measurement of pain, function and ability amongst individuals with LBP should include the use of validated clinical questionnaires to determine the effectiveness of an intervention or rehabilitation programme. Examples of functional assessment include; the Roland Morris Disability Questionnaire, the Core Outcome Measures Index or the Oswestry Disability Index for function and quality of activities of daily living. In addition, assessments of pain frequently reported include; the Numerical Pain Rating Scale (NPRS) and Short Form McGill Pain Questionnaire 2 (SF-MPQ-2) $(15,16,17,18,19)$. The NPRS provides an insight into the subjective severity of pain on a numerical scale from 0 to 10 . The SF-MPQ-2 further investigates the severity of different sub-types of pain (continuous, intermittent, neurological, and affective) on a numerical scale similar to the NPRS. Pain related questionnaires should be used prior to any intervention as a baseline measure, and then again at subsequent follow up sessions to monitor any change in pain. The NPRS has been well researched and a Minimal Clinical Important Difference (MCID) of 1.7 points, or a $28 \%$ change is required to determine that an intervention is useful amongst chronic LBP patients.

However, the presence of pain alone does not give enough information to enable a treatment strategy to be determined, current function and activity levels or limitations should also be considered.

Rehabilitative measures may involve the use of medical devices, such as lumbar bracing to improve alignment and associated pain (20). But may also include simple lifestyle changes such as changing the sleeping surface to a more supportive mattress $(21,22)$, or the introduction of a correctly fitted and supportive bra (23), which can provide important changes in alignment. Postural assessments and corrective techniques are therefore often key to the management of such patients. Malalignment of the spine undoubtedly has a negative impact on the musculoskeletal system. Posture, and therefore spinal alignment, is often assessed by a clinician applying theoretical knowledge through visual assessment. However, more recent complex biomechanical methods have been used to quantify changes in spinal alignment (24), which can be used to determine the efficacy and effectiveness of different treatment interventions (21).

Strength deficits are also often present in LBP patients, which are often unilateral, and result in muscle imbalances in the paraspinal muscles (25). Compensating for muscle imbalances over a prolonged period of time can lead to a multitude of negative health implications (26). In patients with LBP, the paraspinal muscles may exhibit structural changes such as musc- 
le fibre atrophy, which can result in pain, spinal instability, asymmetry and limited range of motion (ROM) (27). Correct spinal alignment is achieved through complex loading patterns on the passive structures of the spine, including the paraspinal and trunk muscles (27). Such activity may be assessed using surface electromyography (EMG), which allows a quantification of the muscle activity around the spine during simple ROM tasks (25). Any imbalance in the muscle activity may be associated with an imbalance in strength which may be directly associated with pain (25).

\section{THE USE OF PASS IN THE MANAGEMENT OF PATELLOFEMORAL PAIN}

Patellofemoral pain (PFP) is a chronic musculoskeletal condition usually presented by persistent pain in, or around, the patella (28). One in five people in the general population experiences PFP (29). However, long term prognosis with current multimodal therapy for PFP is poor (30). A recent paper by Selfe et al (31) explored the use of specific assessments to determine subgroups within the PFP population. These consisted of "weak and pronated" (39\%), "weak and tighter" (39\%), and "strong" (22\%). These subgroups raise a number of important questions about possible treatment strategies. Namely, the use of strengthening protocols in the weak groups, the use of foot orthoses to correct alignment in the weak and pronated group, and the consideration that patellofemoral overload and/or instability may be relevant in the strong group.

All people with PFP present with pain, which can be measured by using the NPRS, VAS, or the newly developed KOOS_PF (32), to set a baseline measurement. Evaluation can happen over time to determine if pain is reduced. The minimal clinical important difference (MCID) for the NPRS and the VAS have been found to be 1 point or $20 \mathrm{~mm}$ in people with chronic musculoskeletal (MSK) pain (18). This means that when a patient shows a change which exceeds this threshold there is a meaningful clinical difference for this patient. A MCID for the KOOS-PF has yet to be determined. However, as with LBP, the presence of pain alone does not give enough information to enable a treatment strategy to be determined.

People in the "weak and pronated" subgroup present with a poor foot position, which in turn can lead to malalignment of the tibia and patella (33). The Foot Posture Index (FPI) (34) is a comprehensive assessment tool to identify foot type. The FPI consists of six measurements that provide a combined score of -12 to 12 . A score over +6 indicates that a patient has a pronated foot (34). Furthermore, a FPI score of +6 was the threshold found for inclusion in the weak and pronated foot group by Selfe et al (31). The alignment of the foot can be corrected using foot orthoses, which in turn can correct the malalignment of the tibia and patella (33).

Individuals with PFP, specifically those within the weak subgroups, most often present with significant differences in the quadriceps femoris muscle (QFM) compared to the healthy population. Differences in the morphology and architecture of the vastus medialis (VMO), particularly in the more distal aspect of the muscle (35) result in under-development and reduced muscle strength compared to healthy individuals (36). In addition, during voluntary muscle contraction, it has been accepted that individuals with PFP present with a delayed muscle activation of VMO compared to vastus lateralis (VL). QFM strengthening, as part of a rehabilitation program for PFP patients, has been supported by Giles et al (37), as it has been identified that QFM atrophy is prevalent amongst PFP patients within the weak subgroups. Neuromuscular electrical stimulation (NMES), has also been shown to improve function and reduces pain amongst Osteoarthritis (OA) patients by targeting the injured/affected structures within the QFM (38). In combination with this, voluntary activation of the QFM is improved, which is an important step within muscle recovery and OA management (39).

Amongst both PFP and OA patients, joint stresses are associated to chondral and osseous changes (40). Through the introduction of NMES, with an aim to improve muscle strength within a PFP rehabilitation program, the functional capacity of QFM may be increased whilst also managing pain, similar to that prescribed for OA patients (41). Dos Santos et al (42) identified that both muscle rebalance and pain relief may be achieved by combining NMES and resistance exercises within an individual PFP patient's treatment plan. Therefore, clinicians may be advised to consider the introduction of NMES and resistance exercises for the treatment for PFP patients within the weak subgroups.

When considering PFP, knee stability and the associated interventions, it is important to look at not just the sagittal plane knee mechanics, but to also consider the movement in the coronal and transverse planes. The tests used to assess movement need to challenge the dynamic control of the patella, however activities such as level walking do not offer a sufficient challenge (42). In addition, Selfe et al (42) described how a dynamic movement such as a step down can give a sufficient challenge to the stability of the knee. Therefore, these dynamic control tests allow for the assessment of stability in not only the sagit- 
tal plane but also the coronal and transverse planes. With the knee having six degrees of freedom of motion, it is important not to ignore motion in the other planes as highlighted by Kowalk et al (43). Kowalk et al (43) showed that the knee abduction-adduction moment should not be ignored when assessing knee stability during stair climbing, even though this is not the primary plane in which motion occurs. In addition, PFP patients who reported the greatest pain have been shown to have the greatest instability (44). Implementing the correct intervention to address this issue of stability is critical. Studies have shown that there are a range of techniques and devices that can offer incremental increases in stability through proprioception and neuromuscular control $(44,45)$. However, it remains unclear whether such effects are present in all of the subgroups identified by Selfe et al (31).

\section{HUMAN FACTORS ASSOCIATED WITH PASS}

Another aspect that should be considered are the human factors, the ways in which a person will interact with the systems around them. This has been acknowledged as an increasingly as a critical part of any product or service design. Indeed, for the first time in 2016, the UK Medicines and Healthcare Regulatory Products Agency (MHRA) released the first draft guidance on human factors aspects of design for medical devices (46). The key principles of human factors are all focused around reducing human error by making a system as simple to use as possible, both cognitively and physically. Is the system easy to use? Where physical products are involved, are they ergonomically sound and comfortable to use? These principles apply for both the patient, and the therapist (47). Therefore, when considering targeted interventions, the PASS framework fits into this by providing therapists with a structured programme to work with, reducing reliance on memory and simplifying the process. For patients, it provides a clear framework for them to understand, aiding acceptance of any intervention.

\section{CONCLUSION}

The clinical assessment and management of musculoskeletal conditions of different joints may be broken down into considerations of Pain, Alignment, Strength and Stability (PASS). This provides therapists with a structured programme to work with, reducing reliance on memory and simplifying the process; whilst for patients, it provides a clear framework for them to understand, aiding acceptance of any intervention.

\section{REFERENCES}

1. Health and Safety Executive. Work related musculoskeletal disorders statistics (WRMSDs) in Great Britain, 2018. Bootle: Health and Safety Executive. 2018 Oct [cited 2018 Nov 18]. Available from: http://www.hse.gov.uk/statistics/causdis/msd.pdf

2. Richards JD, Sanchez-Ballester J, Jones RK, Darke $\mathrm{N}$, Livingstone BN. A comparison of knee braces during walking for the treatment of osteoarthritis of the medial compartment of the knee. The Journal of bone and joint surgery. British volume. 2005; 87(7):937-9.

3. Wearing SC, Hennig EM, Byrne NM, Steele JR, Hills AP. Musculoskeletal disorders associated with obesity: a biomechanical perspective. Obesity reviews. 2006; 7(3):239-50.

4. Craig P, Dieppe P, Macintyre S, Michie S, Nazareth I, Petticrew M. Developing and evaluating complex interventions: The new Medical Research Council guidance.2019; Accessed 06 October 2017. Available from https://mrc.ukri.org/documents/pdf/ complex-interventions-guidance/

5. Kent PM, Keating JL. The epidemiology of low back pain in primary care. Chiropractic \& Osteopathy. 2005; 13(1):13.

6. Comerford MJ, Mottram SL. Functional stability re-training: principles and strategies for managing mechanical dysfunction. Manual Therapy. 2001; 6(1):3-14.

7. Jensen DV, Wormsley M, Minck H, Johansen S. Pregnancy associated pelvic pain II: Symptoms and clinical findings. Ugeskrift for Laeger. 2000; 162: 4813-481

8. Noren L, Ostgaard S, Johansson G, Ostgaard HC. Lumbar back and posterior pelvic pain during pregnancy: a 3-year follow up. European Spine Journal. 2002; 11:267-271

9. Wang SM, Dezinno P, Maranetsvl-Berman MR, Caldwell-Andrews AA. Low back pain during pregnancy: prevalence risk factors, and outcomes. Obstetrics and Gynecology. 2004; 104: 65-70.

10. Manchikanti L, Singh V, Falco F. Epidemiology of Low Back Pain in Adults. Neuromodulation.2014; 17(2): 3-10.

11. Marin R, Cyhan T, Miklos W. Sleep disturbance in patients with chronic back pain. American Journal of Physical Medicine and Rehabilitation. 2006; 85(5): 430-435

12. Andersson GBJ. (1999). Epidemiological features of chronic low-back pain. The Lancet. 1999; 354(9178): 581-585 
13. Liebenson C. Rehabilitation of the Spine. Baltimore: Williams and Wilkins 1996.

14. Mandiakis N, Gray A. The economic burden of low back pain in the United Kingdom, Pain. 2000; 84(1):95-103.

15. Deyo R, Battie M, Buerskens A. Outcome measures for low back pain research: a proposal for standardized use. Spine. 1998; 23: 2003-13

16. Fairbank J, Pynsent P. The Oswestry Disability Index. Spine. 2000; 25(22): 2940-2953

17. Ostello R, Deyo R, Stratford P. Interpreting change scores for pain and functional status in Low Back Pain. Towards International Consensus regarding minimal important change. Spine. 2008; 33(1): 90-94

18. Salaffi F, Stancati A, Silvestri CA, Ciapetti A, Grassi W. Minimal clinically important changes in chronic musculoskeletal pain intensity measured on a numerical rating scale. European Journal of Pain. 2012; 8(4):283-291.

19. Kamper et al, 2010

20. Weiss HR, Werkmann M. Treatment of chronic low back pain in patients with spinal deformities using a sagittal re-alignment brace. Scoliosis. 2009; 9; $4: 7$

21. Chohan A, Payne K, Selfe J, Richards J. Biomechanical testing of a spinal alignment cushion ("Rophi" cushion) in adults with lower back pain. Gait \&Posture. 2013; 38 (Suppl 1), pS104.

22. Chohan A, Shore H, Smith L, Richards, J. Does mattress zoning affect the biomechanics of sleep. 8th World Congress of Biomechanics. 2018; P4221

23. Chohan A, Haworth L, Janssen J, Selfe J. The impact of different breast support garments on larger breasted women with non-specific back pain. Bone and Joint. 2016; 98(B) Suppl 6-13

24. Preuss, RA, Popovic, MR. Three-dimensional spine kinematics during multidirectional, targetdirected trunk movement in sitting. Journal of Electromyography and Kinesiology. 2010; 20(5), pp. 823-832.

25. Oddsson L, De Luca, C. Activation imbalances in lumbar spine muscles in the presence of chronic low back pain. Journal of Applied Physiology. 2003; 94(4):1410-1420.

26. Touche R, Escalante K, Linares M. Treating non -specific chronic low back pain through the Pilates Method. Journal of Bodywork and Movement Therapies. 2008; 12(4), 364-370.

27. Arokoski J, Valta T, Kankaanpää M, \& Airaksinen O. Activation of lumbar paraspinal and abdominal muscles during therapeutic exercises in chronic low back pain patients. Archives of Physical Medicine and Rehabilitation. 2004; 85(5):823-832.

28. Callaghan MJ, Selfe J. Has the incidence or prevalence of patellofemoral pain in the general population in the United Kingdom been properly evaluated?. Physical Therapy in Sport. 2007; 8(1):37-43.

29. Smith BE, Selfe J, Thacker D, Hendrick P, Bateman M, Moffatt F, Rathleff MS, Smith TO, Logan P. Incidence and prevalence of patellofemoral pain: A systematic review and meta-analysis. PLOS ONE. 2018; 11;13(1):e0190892.

30. Lankhorst, N.E., van Middelkoop, M., Crossley, K.M., Bierma-Zeinstra, S.M.A., Oei, E.H.G., Vicenzino, B. and Collins, NJ. Factors that predict a poor outcome 5-8 years after the diagnosis of patellofemoral pain: a multicentre observational analysis. British Journal of Sports Medicine. 2016; 50(14):881-886.

31. Selfe J, Janssen J, Callaghan M, Witvrouw E, Sutton C, Richards J, Stokes M, Martin D, Dixon J, Hogarth R, Baltzopoulos V, Ritchie E, Arden N, Dey P. Are there three main subgroups within the patellofemoral pain population? A detailed characterisation study of 127 patients to help develop targeted intervention (TIPPs). British Journal of Sports Medicine. 2016; 50(14):873-80.

32. Crossley KM, Macri EM, Cowan SM, Collins NJ, Roos EM. The patellofemoral pain and osteoarthritis subscale of the KOOS (KOOS-PF): development and validation using the COSMIN checklist. British Journal of Sports Medicine. 2018; 52(17):1130-1136.

33. Curren, S. In: Self J, Janssen J, Callaghan M, editors. Patellofemoral Pain: An evidence-based clinical guide. New York: Nova Biomedical, 2017; ISBN 978-1-53611-798-1.

34. Redmond AC, Crosbie J, Ouvrier RA. Development and validation of a novel rating system for scoring standing foot posture: The Foot Posture Index. Clinical Biomechanics. 2006; 21(1):89-98.

35. Pattyn E, Verdonk P, Steyaert A, Vanden Bossche L, Van den Broecke W, Thijs Y, Witvrouw E. Vastus Medialis Obliquus Atrophy: Does It Exist in Patellofemoral Pain Syndrome?. The American Journal of Sports Medicine. 2011; 39(7):1450-1455.

36. Van Tiggelen D, Cowan S, Coorevits P, Duvigneaud N, Witvrouw E. Delayed Vastus Medialis Obliquus to Vastus Lateralis Onset Timing Contributes to the Development of Patellofemoral Pain in Previously Healthy Men: A Prospective Study. 
The American Journal of Sports Medicine. 2009; 37(6):1099-1105.

37. Giles LS, Webster KE, McClelland JA, Cook J. Does Quadriceps Atrophy Exist in Individuals With $\mathrm{Pa}$ tellofemoral Pain? A Systematic Literature Review With Meta-analysis. Journal of Orthopaedic \& Sports Physical Therapy. 2013; 43(11):766-76.

38. de Oliveira Melo M, Aragão FA, Vaz MA. Neuromuscular electrical stimulation for muscle strengthening in eldery with knee osteoarthritis A systematic review. Complementary Therapies in Clinical Practice. 2013; 19(1):27-31.

39. Elboim-Gabyzon M, Rozen N, Laufer Y. Quadriceps femoris muscle fatigue in patients with knee osteoarthritis. Clinical Interventions in Aging. 2013; 8:1071-7.

40. Wyndow N, Collins N, Vicenzino B, Tucker K, Crossley K. Is There a Biomechanical Link Between Patellofemoral Pain and Osteoarthritis? A Narrative Review. Sports Medicine. 2016; 46(12):17971808.

41. Dos Santos RL, Souza MMLSP, Santos FA, dos Santo FA. Neuromuscular electric stimulation in patellofemoral dysfunction: literature review. Acta Ortopedica Brasileira. 2013; 21(1):52-58.

42. Selfe J, Richards J, Thewlis D, Kilmurray S. The biomechanics of step descent under different treatment modalities used in patellofemoral pain. Gait \& Posture. 2008; 27(2):258-263.

43. Kowalk DL, Duncan JA, Vaughan CL. Abduction -adduction moments at the knee during stair ascent and descent. Journal of Biomechanics. 1996; 29(3):383-388.

44. Selfe J, Thewlis D, Hill S, Whitaker J, Sutton C, Richards J. A clinical study of the biomechanics of step descent using different treatment modalities for patellofemoral pain. Gait and Posture. 2011; 34(1):92-96.

45. Petersen W, Ellermann A, Gösele-Koppenburg A, Best R, Rembitzki IV, Brüggemann GP, Liebau C. Patellofemoral pain syndrome. Knee Surgery, Sports Traumatology, Arthroscopy. 2014; 22(10):2264-2274.

46. Medicines and Healthcare Products Regulatory Agency. Human Factors and Usability Engineering - Guidance for Medical Devices Including Drug-device Combination Products. London: MHRA. 2017 Sep [cited 2018 Oct 06]. Available from https://assets.publishing.service.gov.uk/government/uploads/system/uploads/attachment_ data/file/645862/HumanFactors_Medical-Devices_v1.0.pdf

47. Health and Safety Executive. Human Factors Principles: Design. Health and Safety Executive, Bootle. [cited 2018 Oct 6]. Available from http://www. hse.gov.uk/humanfactors/topics/design.htm

\section{CORRESPONDENCE ADDRESS OF MAIN AUTHOR}

Alex Mbuli

Sport, Exercise \& Health Sciences

Edinburgh Napier University

Edinburgh, UK

$+447988770838$

email: a.mbuli@napier.ac.uk

Přijato k recenzi: 25. 10. 2018

Přijato do tisku: 12. 12. 2018 